\title{
Local and virtual RBF meshless method for high-speed flows
}

\author{
S. Gerace ${ }^{1}$, K. Erhart ${ }^{1}$, E. Divo ${ }^{2}$ \& A. Kassab ${ }^{2}$ \\ ${ }^{1}$ Convergent Modeling, Inc., USA \\ ${ }^{2}$ Department of Mechanical, Materials, and Aerospace Engineering, \\ University of Central Florida, USA
}

\begin{abstract}
Existing Computational Fluid Dynamic solutions suffer from several major limitations that prevent widespread use within the scope of many complex aerospace flow situations. These limitations include requiring significant model setup time, requiring skilled labor to generate the underlying computational grid, and requiring extensive computational resources to construct large-scale models using conventional techniques. In response to these issues, this work has developed a solution utilizing a novel Meshless method that eliminates the need for structured meshes, and thus, the need for complicated meshing procedures that demand both time and labor to complete. The presented Meshless method, which is based upon two collocation techniques, Local Radial Basis Function (LRBF) collocation and Virtual Radial Basis Function (VRBF) collocation, has shown promising results within the areas of heat transfer and elasticity, as well as incompressible and compressible fluid flow. Incorporation of an automated refinement process based on boundary and interior values provides the method with a high level of robustness with respect to initial point distribution. Additionally, the inclusion of shadow nodes in near-boundary regions allows the method to accurately capture the high gradients present in typical high-speed boundary layers. Several examples are presented within the area of high-speed flow in an attempt to highlight the ease of use, as well as the accuracy of the described techniques.

Keywords: meshless methods, radial basis functions, generalized finite differencing, CFD, Navier-Stokes.
\end{abstract}




\section{Introduction}

As compared to traditional, mesh-based solution techniques, the so-called Meshless, or mesh-reduction methods promise to eliminate the tedious mesh-generation process by improving the robustness of the algorithm with respect to the underlying point distribution. This mesh-generation process is currently a major requirement of time and effort when attempting to solve problems involving complex geometries where automatic Finite Element or Finite Volume meshes provide unacceptable solution quality.

Through presentation of both the underlying theory and basic algorithm structure, we attempt to illustrate the advantages of the presented Meshless method over more conventional techniques. Throughout the development of these techniques, focus was placed on accuracy as well as automation, allowing for a highly user friendly CFD solution. Several test cases with comparisons to experimental data, as well as to existing commercially available CFD technology are presented as evidence supporting the adoption of this method as a viable alternative to more conventional CFD approaches.

\section{Meshless collocation techniques}

Conventional numerical methods commonly used in most engineering applications (Finite Element, Finite Volume, and to some degree, Boundary Element methods) all introduce the idea of a defined connectivity between nodes or volumes. This connectivity is what allows the various techniques to determine the influence of any node to its neighbors. While it is true that for simple models, development of this connectivity can be largely automated, as the complexity and size of the problem increases, it becomes exceedingly difficult to automate this procedure. With Meshless methods, on the other hand, the underlying goal is to eliminate this need for a defined connectivity mesh. Instead, the influence of one node on its neighbors is defined by an interpolation technique that can be used regardless of model geometry or nodal spacing.

Although many interpolation techniques exist that may be used to arrive at a Meshless formulation, this paper will present the two specific techniques that have shown promising results within the area of high-speed flow.

\subsection{Localized radial basis function collocation}

Localized Radial Basis Function collocation begins with the principle that any arbitrary domain, $\Omega$, can be interpolated over by collocating about a number of points using some basis function, $\chi$. This method (and in fact, both Meshless collocation techniques implemented in this paper) divides the overall region into smaller sub-domains, called topologies, which allow for a more efficient and accurate solution method when compared to global interpolation techniques. As a simple example, the process of breaking down a two-dimensional region into representative topologies is demonstrated in Figure 1(a). 


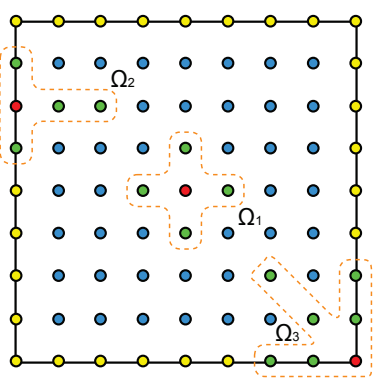

(a) Representative 2D Domain

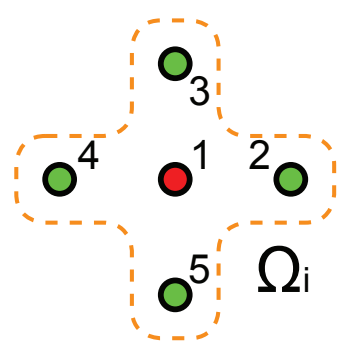

(b) Representative 2D Topology

Figure 1: Localized topology representation.

The region shown is a two-dimensional representation, however, in general, the nodes can describe an $n$-dimensional field (where $n$ is generally 2 or 3 ). The represented field, $\phi$, can be shown to be globally interpolated [1] by multiplying the basis functions by a set of expansion coefficients as

$$
\phi(x)=\sum_{j=1}^{N} \alpha_{j} \chi_{j}(x)+\sum_{k=1}^{N P} \alpha_{(k+N)} P_{k}(x)
$$

where $N$ is the total number of points in the domain, $\alpha_{j}$ are the expansion coefficients for $\phi$, and $\chi_{j}(x)$ are a-priori defined expansion functions. Additionally, a similar expansion is performed over $N P$ polynomial functions (or any additional expansion function), $P_{k}(x)$, which must be added to the overall expansion to guarantee that constant and linear fields can be retrieved exactly [2]. However, this formulation assumes a global collocation, which, as already stated, is not ideal. Thus, the concept of local topologies can be used to reformulate Eq. (1) such that instead of summing over the entire domain, the basis function is now applied to the local topologies, $\Omega_{i}$. Therefore, the locally interpolated field may be expressed as

$$
\phi(x)=\sum_{j=1}^{N F} \alpha_{j} \chi_{j}(x)+\sum_{k=1}^{N P} \alpha_{(k+N F)} P_{k}(x)
$$

where instead of summing over the entire region, Eq. (2) is instead summed over the number of points in a given topology, expressed as $N F$. For example, looking at the two-dimensional representative topology shown in Figure 1(b), $N F$ is equal to 5 , as there are 5 nodes included in the topology for the data center (Node 1).

A critical component to this type of Meshless collocation technique is determining a suitable basis function, $\chi$, that will accurately interpolate between data points. Much research has gone into analyzing the behavior of the most common basis functions for this type of technique [2,3], and the most accurate and stable was determined to be the family of so-called Inverse Hardy Multiquadrics [4] (an 
Inverse Multiquadric function where $n=1$ ) which follows the form

$$
\chi\left(x_{i}\right)=\frac{1}{\sqrt{r_{j}\left(x_{i}\right)^{2}-c^{2}}}
$$

where $x$ is the position vector at a given location $i, r_{j}$ is the Euclidean distance from point $i$ to point $j$, and $c$ is a free constant commonly referred to as the basis shape parameter. Optimizing this shape parameter is critical to obtaining accurate results and fortunately our research group has developed a very novel method of arriving at an optimal value on a topology by topology basis by means of Singular Value Decomposition [2,5]. Thus, this expansion function $\phi(x)$ is used to locally interpolate the field about a surrounding node, providing the "connectivity" (more appropriately referred to as influence) required to evaluate the necessary derivatives of the field.

Having defined the interpolation method used in this collocation technique, the next step is to construct the weights associated with the derivative operators. To accomplish this, Eq. (2) may be applied to all nodes within a topology, which results in a field interpolation within each local topology region; however, it may also be differentiated, thus providing a means of evaluating derivative values. Thus, to represent a particular derivative of the field $\phi$,

$$
\partial \phi(x)=\sum_{j=1}^{N F} \alpha_{j} \partial \chi_{j}(x)+\sum_{k=1}^{N P} \alpha_{(k+N F)} \partial P_{k}(x)
$$

where $\partial$ may represent any differential operator. For example, the Laplacian operator would be evaluated as

$$
\nabla^{2} \phi(x)=\sum_{j=1}^{N F} \alpha_{j} \nabla^{2} \chi_{j}(x)+\sum_{k=1}^{N P} \alpha_{(k+N F)} \nabla^{2} P_{k}(x)
$$

Although the Localized RBF collocation process provides adequate numerical results in most cases, it suffers from several key issues which prevent it from being used in all cases:

1. Since the basis functions were chosen to be radially symmetric, optimal results are only obtained for radially symmetric derivative operators. Onesided operators (such as upwinded derivatives) are not represented well by the radial nature of the interpolator.

2. In areas consisting of locally structured nodes, it can be shown that the weights obtained through this process are identical to those obtained through traditional finite differencing; we would like to take advantage of this case without having to compute unnecessary quantities.

3. Areas of very high gradients (shocks, boundary layers, etc.) may cause oscillations in the RBF interpolator, resulting in poor numerical stability.

In response to these issues, a second collocation technique is utilized and is applied at areas with local structure, and for those derivative operators that are 


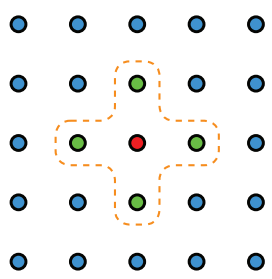

(a) Structured Topology

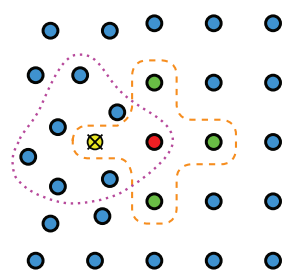

(b) Unstructured Topology

Figure 2: Structured and unstructured meshless topologies.

not symmetric. Additionally, if an RBF interpolation value is deemed oscillatory in nature (by looking at the local field values), it is (temporarily) replaced with the second type of collocation technique to assist in numerical stability.

\subsection{Virtual radial basis function collocation}

Conventional Finite Difference collocation techniques involve truncating the Taylor series expansion to approximate a given derivative at a specific location within a field. The Finite Difference formulations can therefore be directly applied to any regular point distribution when the surrounding nodes are properly located within the bounds of the approximation. However, this technique has a limitation in that it requires a regular, defined distribution of nodes, something that is not possible for an unstructured, Meshless domain. By utilizing some of the concepts of Localized Radial Basis collocation, the standard Finite Difference formulation may be extended to non-regular nodal distributions and be made into a Meshless technique.

Virtual Radial Basis Function (VRBF) collocation departs from the standard Finite Difference formulations for the required derivatives, including all necessary upwinding states. In the case of locally structured regions, such as depicted in Figure 2(a), the Finite Difference formulations may be directly applied. Although this is a Meshless technique, we still attempt to create as much structured region as possible (by utilizing an octree point distribution method) to provide the maximum accuracy and performance of the algorithm; as such, a large portion of the domain is usually structured. For those regions that are not completely structured, such as depicted in Figure 2(b), additional, virtual nodes are placed in the necessary locations (in this case, in the negative $x$ direction, indicated by the yellow $\otimes$ ) in order to facilitate use of the Finite Difference equations. However, since this is a virtual node that does not actually exist (and thus, no governing equations are solved), we require a means of evaluating the field at the virtual node location. To accomplish this, a topology is constructed around about this node (purple dotted region), and LRBF is used to interpolate to the virtual location. Thus, despite there being no node at a necessary Finite Difference point, we may still apply these equations due to the interpolation capabilities of the Localized Radial Basis Function collocation method. 


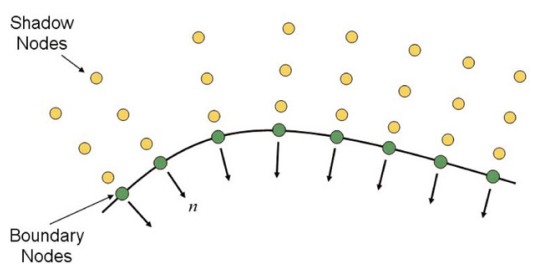

(a) Convex Boundary Layer

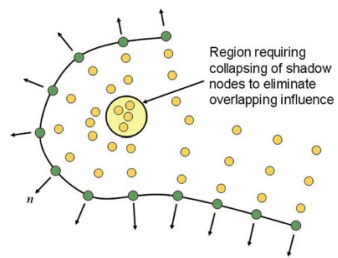

(b) Concave Boundary Layer

Figure 3: Illustration of shadow nodes.

\section{Model construction and refinement}

One of the critical advantages of Meshless methods is allowing for the capability of completely automated point and topology generation in arbitrarily complex three dimensional models. Therefore, we have spent considerable efforts toward the development of techniques for automated nodal distributions as well as solutionbased adaptive refinement of these discretizations.

Our models are first constructed from a triangulated surface which defines the boundary of the problem. Triangulated, or tessellated, volumes were chosen for several reasons. First, because the tessellated volume is a very common entity in computer geometry and graphics, extensive literature exists describing how best to control refinement on the surface. Second, because the triangulated surfaces are relatively simple compared to other analytical surfaces, calculations required for surface integrations, volume calculations (volume, centroid, containment tests), and other necessary components, are relatively straightforward to implement and fast in calculation. Third, even though solutions may be obtained using Meshless methods without a defined boundary connectivity, in order to post process terms such as surface forces, stresses, moments, and other area based values, each boundary node must have a defined area and normal direction. By using a triangulated surface representation, the boundary nodes inherit their parent facet's area and normal properties, facilitating easy translation from the Meshless solution domain into the geometric domain of the problem.

Once the boundary has been discretized to a sufficient level, the next step in the point distribution process is to create the so-called "shadow" nodes, which serve to function as a boundary layer distribution in the problem solution. The process of adding shadow nodes is shown for a simple two dimensional boundary in Figure 3(a).

It is important to realize that this process of adding shadow nodes can potentially introduce problems in highly convex or concave boundary situations (an issue which is compounded in three dimensions). For the case of convex boundaries (like that shown in Figure 3(a)) any gaps may still be filled with interior nodes once the octree distribution is applied. The rules governing interior node placement do not preclude nodes from entering gaps in the boundary layer. For the case of highly concave boundaries (like that shown in Figure 3(b)) a technique of collapsing 


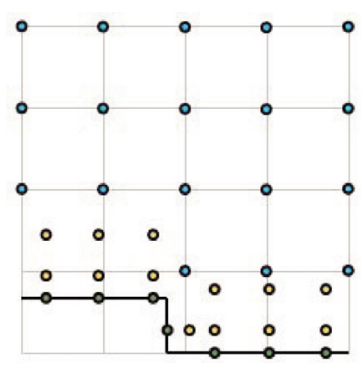

(a) Before Boundary Refinement

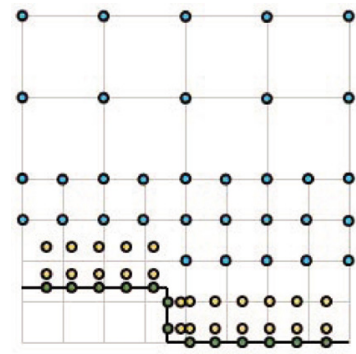

(b) After Boundary Refinement

Figure 4: Sample boundary interior refinement.

shadow nodes which are very close to one another is necessary in order to eliminate instability in the underlying Meshless interpolations.

The collapsing process entails producing an interpolation sphere about all of the shadow nodes affected, and "lumping" their combined values into a single common location. This lumping process is achieved by the Radial Basis Function interpolation that is used throughout the Meshless process. Although each node will still act as a single entity and the solution is still solved at each node independently, to all the other nodes (ones that are not part of the sphere) the cluster appears as a single node.

The final development in the point distribution process is to construct the recursive octree structure used throughout the bulk of the domain interior. An octree structure is used for several reasons, the most important being its ability to refine itself in a very straightforward and automated fashion. It is important to realize that the refinement of the interior, shadow, and boundary nodes is inherently a disconnected process, so compatibility conditions must be included in order properly marry the refinements. We have implemented a process where, as one local region is refined, all other regions which exist in this nearby area will be refined as well. Take, for example, the situation shown in Figure 4. In Figure 4(a), we see an initial discretization for part of a two dimensional boundary. After some number of iterations, it is determined that the current discretization at the boundary layer (yellow shadow nodes) is not sufficient to accurately capture the high gradients that were detected. Rather than simply refine only the boundary nodes (green nodes) and their associated shadow nodes, the interior octree distribution (blue nodes) knows to refine to an appropriate level to match the nearby boundary discretization, as shown in Figure 4(b).

It is important to note that this refinement can begin anywhere within the region (boundary, shadow or interior nodes) and will propagate to all nearby regions. So, for example, a wake zone occurring completely within the interior may still be refined, even though it is not close to any boundary zones. 


\section{Governing equations}

The current Meshless formulation begins with the three-dimensional NavierStokes equations in conservative variable form, given in vector form as

$$
\frac{\partial Q}{\partial t}+\frac{\partial E}{\partial x}+\frac{\partial F}{\partial y}+\frac{\partial G}{\partial z}=\frac{\partial E_{v}}{\partial x}+\frac{\partial F_{v}}{\partial x}+\frac{\partial G_{v}}{\partial x}
$$

where

$$
\begin{gathered}
Q=\left[\begin{array}{c}
\rho \\
\rho u \\
\rho v \\
\rho w \\
\rho e_{t}
\end{array}\right] \quad E=\left[\begin{array}{c}
\rho u \\
\rho u^{2}+p \\
\rho u v \\
\rho u w \\
\left(\rho e_{t}+p\right) u
\end{array}\right] \quad F=\left[\begin{array}{c}
\rho v \\
\rho u v \\
\rho v^{2}+p \\
\rho v w \\
\left(\rho e_{t}+p\right) v
\end{array}\right] \quad G=\left[\begin{array}{c}
\rho w \\
\rho w u \\
\rho w v \\
\rho w^{2}+p \\
\left(\rho e_{t}+p\right) w
\end{array}\right] \\
E_{v}=\left[\begin{array}{c}
0 \\
\tau_{x x} \\
\tau_{x y} \\
\tau_{x z} \\
E_{v}^{\prime}
\end{array}\right] F_{v}=\left[\begin{array}{c}
0 \\
\tau_{y x} \\
\tau_{y y} \\
\tau_{y z} \\
F_{v}^{\prime}
\end{array}\right] G_{v}=\left[\begin{array}{c}
0 \\
\tau_{z x} \\
\tau_{z y} \\
\tau_{z z} \\
G_{v}^{\prime}
\end{array}\right]
\end{gathered}
$$

and $E_{v}^{\prime}=u \tau_{x x}+v \tau_{x y}+w \tau_{x z}-q_{x}, F_{v}^{\prime}=u \tau_{y x}+v \tau_{y y}+w \tau_{y z}-q_{y}$, and $G_{v}^{\prime}=u \tau_{z x}+v \tau_{z y}+w \tau_{z z}-q_{z}$. Note that Eq. (6) represents the full Navier-Stokes equations, where $E, F$, and $G$ represent the convective terms of the governing equations and $E_{v}, F_{v}$, and $G_{v}$ represent the viscous terms. Additionally, the shear stress component $\tau_{i j}$ may be expressed in vector form as

$$
\tau_{i j}=\mu\left(\frac{\partial u_{i}}{\partial x_{j}}+\frac{\partial u_{j}}{\partial x_{i}}\right)-\delta_{i j} \frac{2}{3} \mu \frac{\partial u_{k}}{\partial x_{k}}
$$

where $\delta_{i j}$ is the Kronecker Delta. Also, in order to arrive at a complete set of equations the ideal gas equation of state $p=\rho R T$ must be imposed.

In order to solve this set of equations our solution approach utilizes a fully explicit time-marching scheme to reach steady-state solutions. It is worth noting that this approach is completely applicable to unsteady flows as long as a suitable initial condition is provided. However, since our current efforts are focused on reaching steady-state solutions in an efficient manner, a local time-stepping procedure has been implemented to expedite convergence. Local time stepping is well described in many resources (such as Hoffman [6]) and is based on maintaining the local CFL number below the stability threshold. The standard explicit time marching scheme allows all unknown field derivatives to be evaluated at the previous time step, thereby creating a very simple update or advancement equation. The required field derivatives however, must be captured in an accurate fashion in order to produce a reliable CFD approach. It is well known that standard central type differencing produces accurate and stable results for the diffusion/stress terms as these terms generally promote changes nearly uniformly in all directions. The 


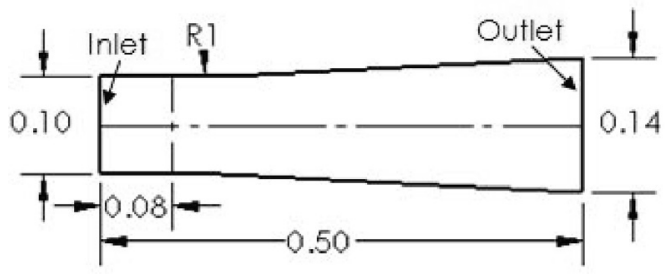

Figure 5: Smooth expanding nozzle geometry (all units in meters).

convective terms however, carry much of the flow information with them so that symmetry in the derivatives is typically never seen. It is this lack of symmetry that produces the need to upwind the convective derivatives in order to ensure the proper direction of travel of the flow field information. The importance of the upwinding procedure is widely known and our Meshless method approach has been found to have similar behavior to that of Finite Differencing in terms of the effects of improper upwinding. For this reason we have implemented a form of the Advection Upstream Splitting Method (AUSM) proposed by Liou and Steffen [7], which seeks to combine the accuracy of the Roe splitting method with the speed of more simplified splitting methods such as the Van Leer Splitting scheme.

\section{Results}

To illustrate the concepts and developments presented in this paper, two test cases will be presented. These test cases were designed to either highlight a particular developmental effort, or to illustrate the overall effectiveness and applicability of our Meshless CFD techniques.

\subsection{Supersonic smooth expanding nozzle}

The first quantitative test case will deal with analyzing viscous flow through a simple smooth expanding nozzle. A two-dimensional depiction of the problem geometry is given in Figure 5, with an understanding that this problem was constant in the $z$ direction, having a domain thickness of $0.05 \mathrm{~m}$.

To generate a supersonic flow field, an inlet Mach number of $M=2$ and a stagnation pressure and temperature of $P_{0}=100000 \mathrm{~Pa}$ and $T_{0}=300 \mathrm{~K}$, respectively, were imposed. Additionally, all non-inlet and outlet walls were assumed to be friction free (slip walls).

This case, due to the steepness of the transition within the nozzle, exhibits a series of interacting compression waves within the nozzle. Although this indicates a poor nozzle design, it serves as an interesting test problem due to the multiple shock interactions which take place within the computational domain. For this particular problem, the Meshless solution began with an initial discretization of approximately 45,000 nodes and was allowed 3 levels of refinement at intervals of 4000 iterations, resulting in a final grid consisting of approximately 160,000 


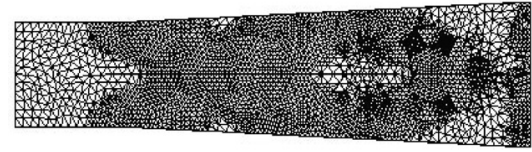

(a) Surface Distribution

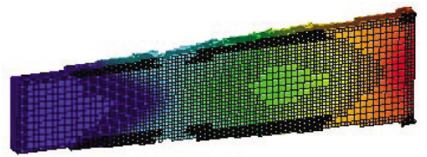

(b) Octree Distribution

Figure 6: Point distributions for supersonic smooth expanding nozzle.

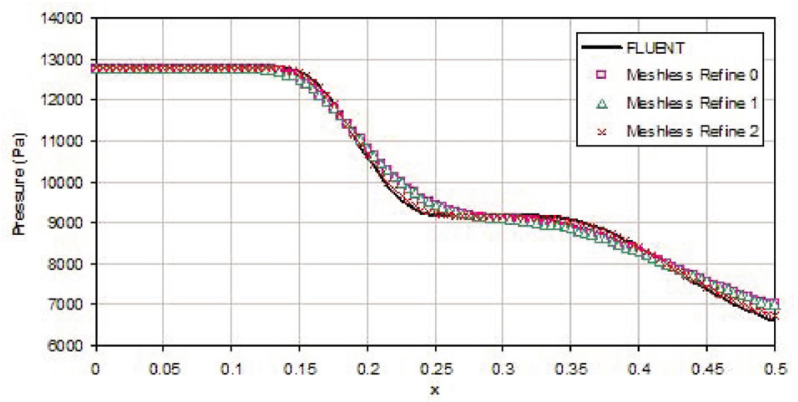

Figure 7: Midline pressure comparison.

nodes. Additionally, the results from this case were compared against a two dimensional solution generated by the commercial CFD package FLUENT, whose computational grid consisted of approximately 70,000 cells (which, in equivalent $3 \mathrm{D}$, would correspond to more than 500,000 cells).

To illustrate the refinement process, Figure 6(a) shows the final surface distribution generated and Figure 6(b) shows a slice exposing the final octree distribution. Note that these point distributions were the result of 3 levels of refinement on both Mach and pressure gradients on the boundary and interior.

For a quantitative comparison, the pressure levels along a mid line ( $y=0, z=$ 0.025) were compared to those obtained via FLUENT and shown in Figure 7, with several Meshless solutions shown, representing the different stages of adaptive refinement. By examining the Meshless results as more points are added, we see that the solution quality is improving as the point distribution is adaptively refined.

Thus, there is excellent agreement between the solutions obtained using FLUENT and the final refined point distribution solved using the proposed Meshless method technique. Additionally, this problem illustrates a major advantage of the proposed technique over other methods in that an initially poor discretization does not prevent the user from obtaining a good final solution. Furthermore, the user need not be aware of any characteristic flow phenomenon prior to obtaining results as the proposed technique is capable of capturing enough of the underlying flow characteristics to allow for proper localized refinement. 


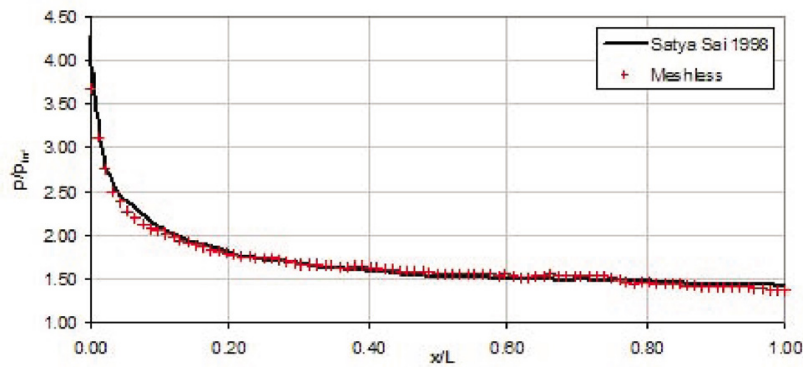

(a) Pressure distribution along plate.

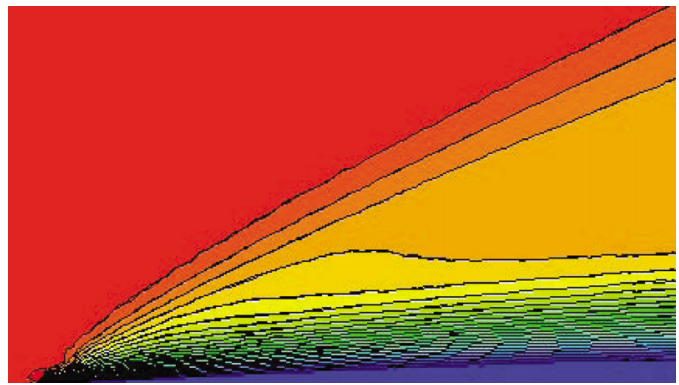

(b) Mach contours.

Figure 8: Supersonic flat plate results.

\subsection{Supersonic flat plate}

In the second test case, a flat plate of unit length was modeled, with the inlet at a distance of 0.2 units from the front of the leading edge and the vertical boundary at a distance of 0.8 units from the flat plate. The plate was therefore assumed to be infinite in length, as the outlet immediately followed the unit length plate. The inlet conditions imposed include a Reynolds number of $R e_{\infty}=1000$, and a Mach number of $M_{\infty}=3.0$. Once again an initial distribution was created and the solution was allowed to refine 3 levels, resulting in a final point distribution consisting of approximately 200,000 nodes. As a comparison, Marshall [8] solved a similar problem with a finite plate whereby the results were verified against those obtained by Satya Sai et al., which detail an infinite plate. As such, the normalized pressure distribution along the length of the flat plate is directly compared to the Satya Sai et al. results quoted by Marshall, and can be seen in Figure 8(a).

Analyzing Figure 8(a), there appears a very good correlation between the results obtained by Satya Sai et al. and the proposed Meshless methods, even in the high pressure region at the onset of the flat plate. To further illustrate the obtained solution, Figure $8 \mathrm{~b}$ shows the obtained Mach contours near the onset of the flat plate. This figure illustrates that the shock wave is being accurately captured, as well as the resulting boundary layer downstream from the beginning of the flat plate. 


\section{Conclusions}

In conclusion, we have presented a localized Meshless method for the solution of compressible, viscous flows utilizing an innovative blend of Localized and Virtual Radial Basis Function collocation techniques. Through implementation of an adaptive point distribution method which is capable of both boundary and interior refinement, we were able to demonstrate promising results for two highspeed flow test cases. In the process, we have illustrated one of the key advantages of this technique over more traditional methods; because of the robustness of the algorithm, even a very rough initial point distribution is capable of arriving at some fundamentally correct solution. Thus, by coupling with an automatic refinement procedure, the operator truly need not have any prior inclination about the underlying flow characteristics in order to arrive at accurate results. Any flow characteristics (such as boundary layers, shocks, etc.) that were not accurately captured in the original point distribution will be automatically detected, properly refined, and captured.

\section{Acknowledgements}

Convergent Modeling, Inc. would like to acknowledge the funding received in support of this project from the Naval Air Systems Command under the STTR topic N08-T008.

\section{References}

[1] Gerace, S., A Meshless Method Approach for Solving Coupled Thermoelasticity Problems. Honors in the Major Thesis, University of Central Florida, 2006.

[2] Kassab, A. \& Divo, E., An efficient localized radial basis function meshless method for fluid flow and conjugate heat transfer. ASME Journal of Heat Transfer, 129, pp. 179-183, 2007.

[3] Divo, E. \& Kassab, A., A meshless method for conjugate heat transfer. Engineering Analysis, 29, pp. 136-149, 2005.

[4] Hardy, R.L., Multiquadric equations of topography and other irregular surfaces. Journal of Geophysical Research, 76, pp. 1905-1915, 1971.

[5] Gerace, S., An Interactive Framework for Meshless Methods Analysis In Computational Mechanics And Thermofluids. Master's thesis, University of Central Florida, 2007.

[6] Hoffmann, K.A. \& Chiang, S.T., Computational Fluid Dynamics, volume 2. Engineering Education System, 4th edition, 2004.

[7] Liou, M.S. \& Steffen, C.J., A new flux splitting scheme. Journal of Computational Physics, 107, pp. 23-39, 1993.

[8] Marshall, D.D., Extending the Functionalities of Cartesian Grid Solvers: Viscous Effects Modeling and MPI Parallelization. Ph.D. thesis, Georgia Institute of Technology, 2002. 